\title{
A Case Study: Effect of Progressive Resistance and Balance Training on Upper Trunk Muscle Strength of Children with Cerebral Palsy
}

\author{
*Mehrnoush Ismailiyan ${ }^{1}$, Seyed Mohammad Marandi ${ }^{2}$, Fahimeh Esfarjany ${ }^{1}$, Alireza Ghardashi Afousi ${ }^{1}$, Ahmadreza Movahedi ${ }^{3}$ \\ 1. Department of Sport Physiology, Faculty of Physical Education, University of Isfahan, Isfahan, Iran. \\ 2. Department of Sport Physiology, Faculty of Physical Education and Sport Sciences, University of Tehran, Tehran, Iran. \\ 3. Department of Motor Behavior and Sport Management, Faculty of Physical Education, University of Isfahan, Isfahan, Iran.
}

dtation: Ismailiyan M, Marandi SM, Ghardashi Afousi AR, Movahedi AR, Esfarjany F. [Effect of progressive resistance and balance training on upper trunk muscle strength of children with cerebral palsy: A case study (Persian)]. Journal of Rehabilitation. 2016; 17(1): 84-93.

http://dx.doi.org/10.20286/jrehab-170182

: http://dx.doi.org/10.20286/jrehab-170182

Received: 11 Jun. 2015 Accepted: 21 Oct. 2015

Keywords:

Resistance training, Muscle strength, Cerebral palsy, Organizational case studies

\section{ABSTRACT}

Objective Non-progressive cerebral palsy (CP) is due to abnormal development of brain and or brain damages before, during, or after birth. The reason of cerebral palsy is brain damage or its abnormal development. Most of these problems happen when the child is in mother's womb, but the chance is high that it happens in the first 2 years of life, when the brain develops. One of the commonest and most important debilitating signs of cerebral palsy is damage to higher functions, in such a way that the control of moves in grasping and leaving get damaged and in the end, the strength of hand for planning of movement decreases. Several studies have examined the effect of resistance training on muscle strength of patients with $\mathrm{CP}$, while none of them has examined the effect of progressive resistance and balance training in children with CP. This study aimed to investigate the effects of progressive resistance and balance training on upper trunk muscle strength of children with CP.

Materials \& Methods Three boys with cerebral palsy (two patients who were 7 years old and one who was 6) participated in this research. In this study, single subject research method with A-B-A plan was used. Progressive resistance and balance training were administered for 3 days per week for 8 weeks. Wrist and elbow flexor muscles strength was measured by PowerTrack Õ dynamometer (manufactured by JTECK with $4.4 \mathrm{~N}$ threshold).

Results According to visual analysis of data diagrams and based on descriptive statistical indexes and visual analyses, the results showed that resistance and balance training in intervention situation compared to baseline increased the strength of elbow flexor (percentages of non-overlapping data for the first and second participants were $75 \%$ and for the third participant, 100\%). The strength of upper trunk muscles after intervention hve improved compared to baseline; however, one month after intervention, the trend was pretty stabilized.

Conclusion The results of this study showed that 8 weeks of progressive resistance and balance training (in combination) has increased muscle strength in children with cerebral palsy. The present research showed that resistance and balanced trainings have significant effects on muscle strength of children with CP. It seems that these practices have been effective, especially for the wrist flexor and elbow flexor muscles. It can be said that the increase in the muscles of children with CP was due to practice principle along with increase in neuronal compatibility. One of the important points in the effectiveness of resistance training is the intensity of training. The results showed that resistance and balanced trainings increase the muscle strength of children with CP. This power could be partly due to increase in muscle volume and partly due to anabolic hormones.

\section{* Corresponding Author:}

Mehrnoosh Esmailiyan, MSc.

Address: Department of Sport Physiology, Faculty of Physical Education, University of Isfahan, Hezar Jarib St., Azadi Sq., Isfahan, Iran. Tel: +98 (937) 6196458

E-Mail: mehrnoosh.esmailiyan@yahoo.com 


\title{
مطالعه موردى: تأثير تمرينات مقاومتى ييشرونده و تعادلى بر قدرت عضلانى بالاتنه كودكان فلج مغزى
}

\author{
"مهرنوش اسماعيليان'، سيدمحمد مرندى'، فهيمه اسفرجانى'، عليرضا قارداشىافوسى'، احمدرضا موحدى' \\ ا - كروه فيزيولوزيى ورزشى، دانشكدة تربيت بدنى، داثشكاه اصفهان، اصفهان، ايران.

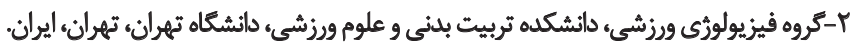

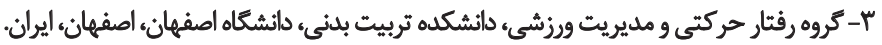

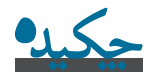

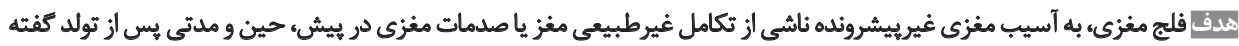

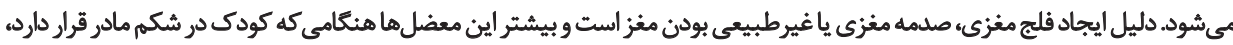

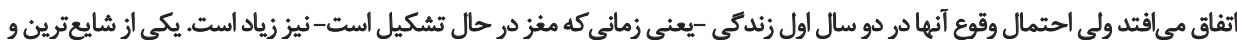

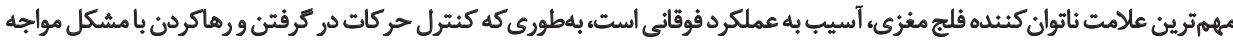

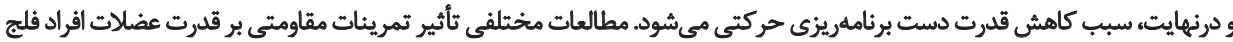

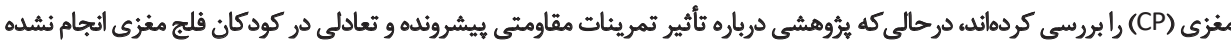

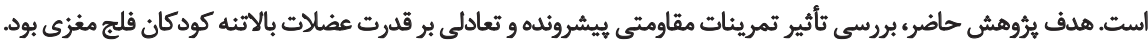

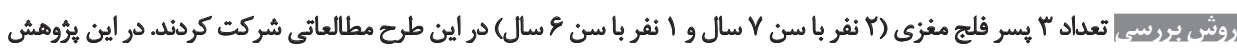

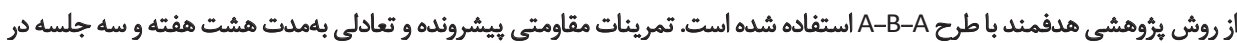

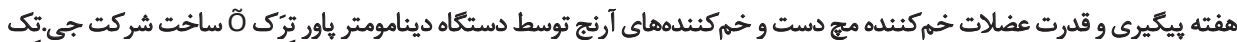

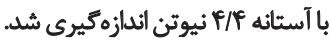

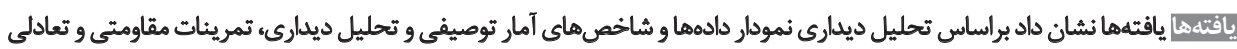

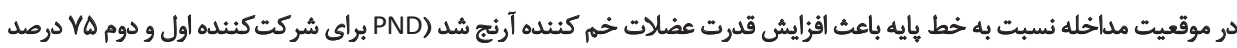

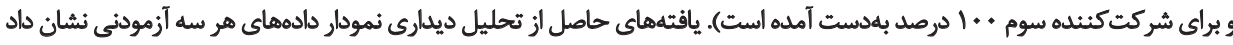

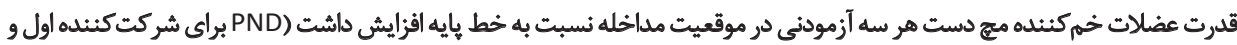

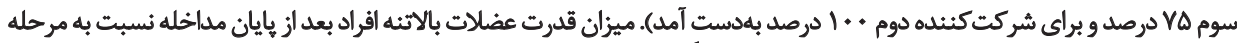

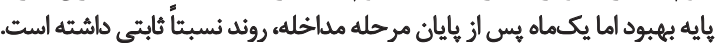

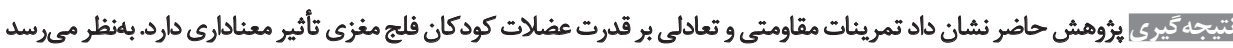

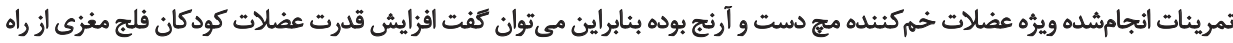

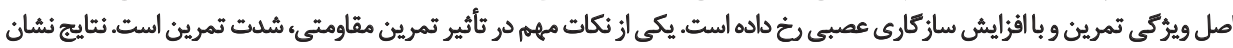

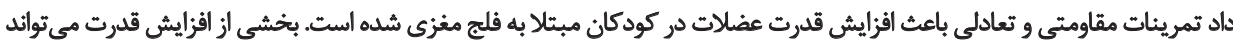

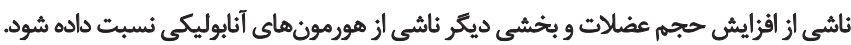

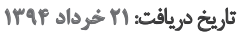

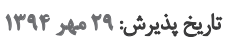

كليدواروهها: تمرين مقاومتى، قدرت عضلانى، فلج مغزي، مقاومي، قدرت مطالعه موردى 


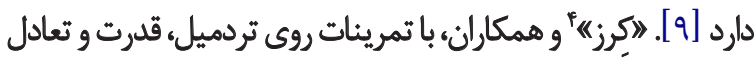

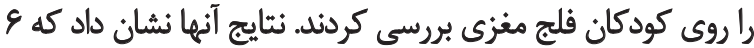

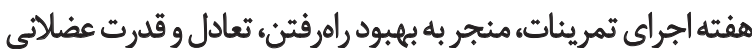

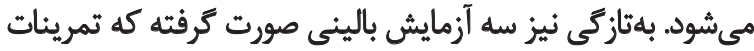

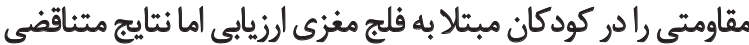

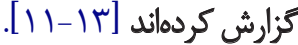

"العبدالوهاب" و همكاران مطالعالى را با هدف تأثير تمرينات

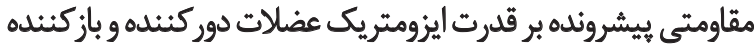

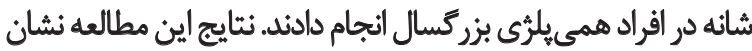

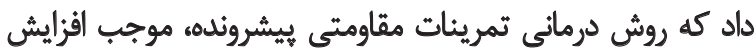

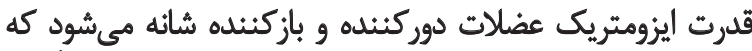

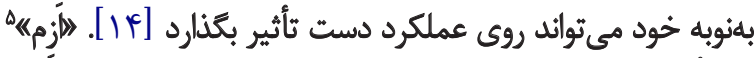

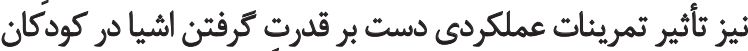

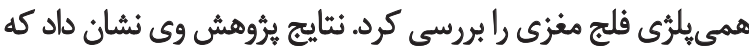

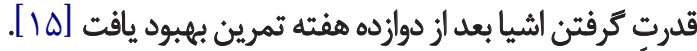

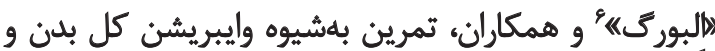

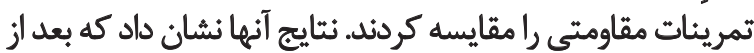

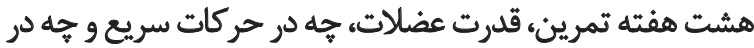

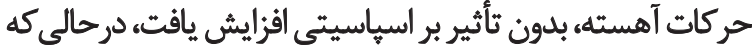

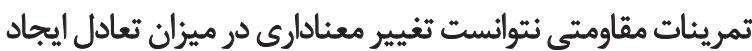

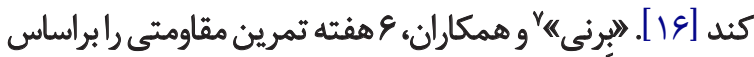

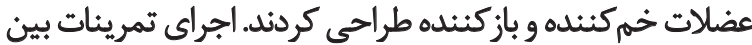

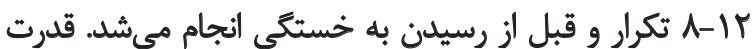

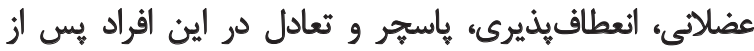

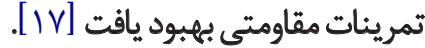

يكى از دلايل تناقض نتايج اين مطالعاته تنوع در ويرگّى نهاى

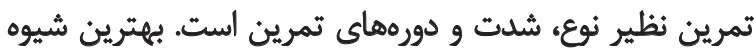

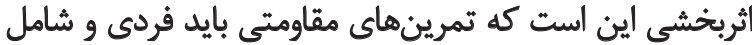

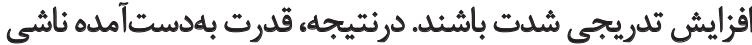

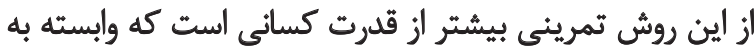

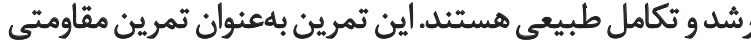

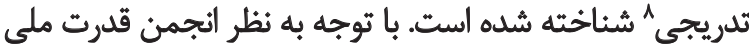

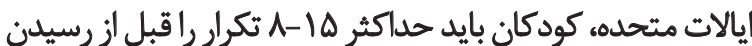

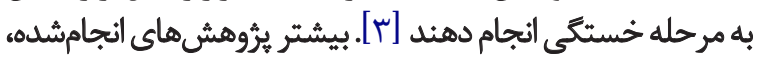

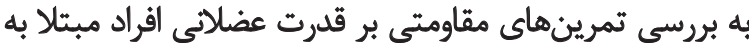

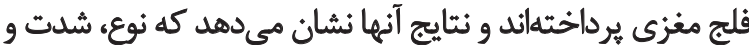

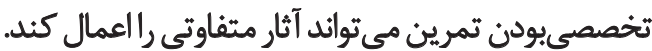

4. Kurz

5. Azzam

6. Ahlborg

7. Burney

8. Progressive resistance exercise

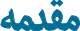

فلج مغزى (CP)'، يك اختلال حركتى ناشي از يك ضايعه

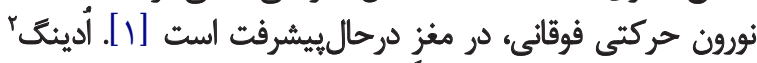

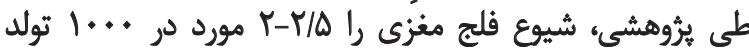

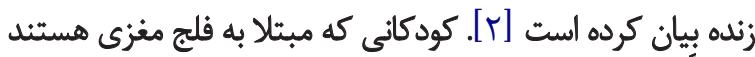

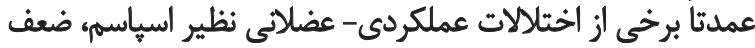

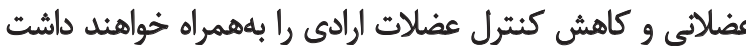

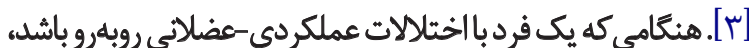

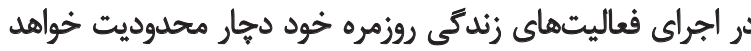

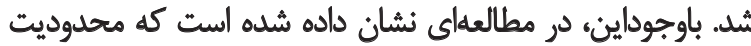

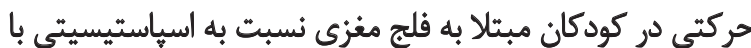

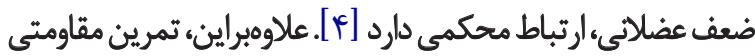

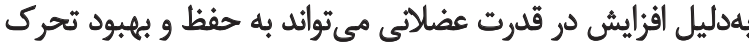
كودكان مبتلا به فلج مغزي كمك نمايد [بآل در سالهاى اخير، تمرين مقاومتى بهعنوان يك مداخله مناسب

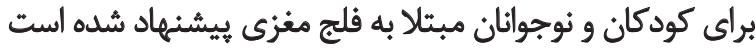

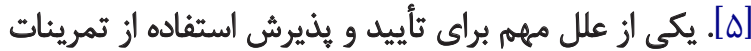

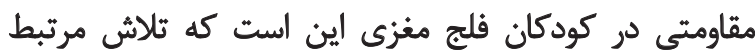

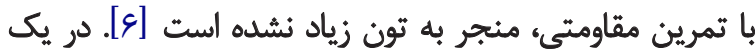

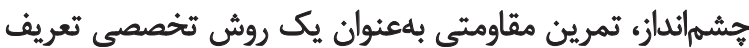

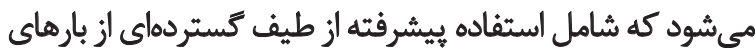

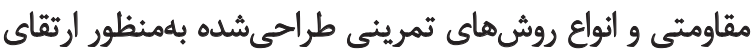

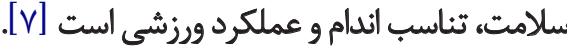

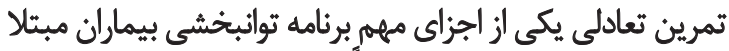

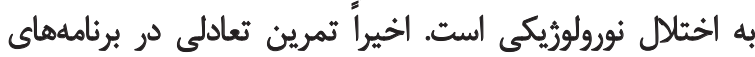

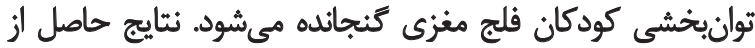

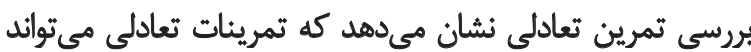

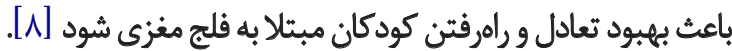
تاجند سال بيش، استفاده از تمرينات مقاومتى در كودكان مبتلابه

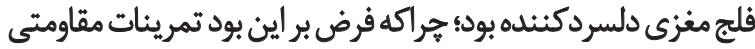

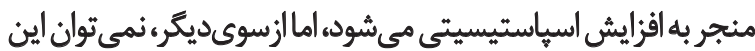

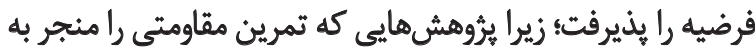

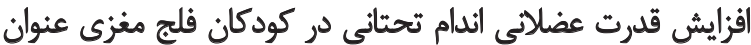

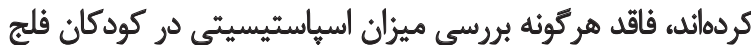

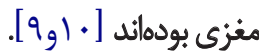
الهورتون" و ومكاران در يك مطالعه كنترلنشده، آثار تمرينات

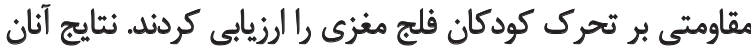

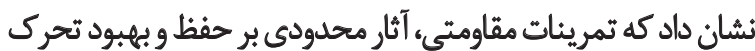

1. Cerebral palsy

2. Odding

3. Morton 
مرحله A2 شامل مر حله بِيعيرى يا مرحله بايه ثانويه است كه شامل

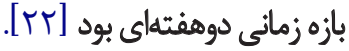

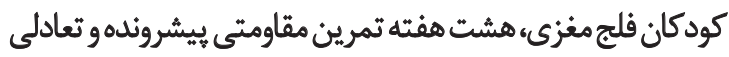

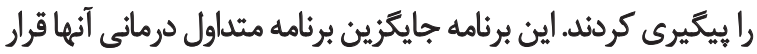

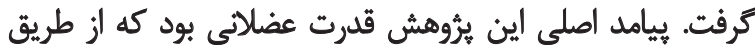

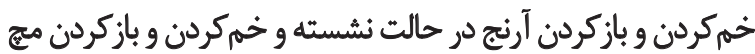
دست بههمراه تمرين تعادلى بهصورت همزمان انجام شدر

طرح تمرين شامل هشت هفته تمرين، سه جلسه در هفته

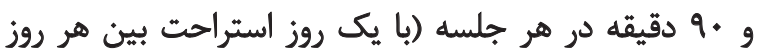

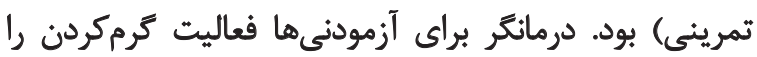

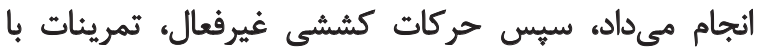

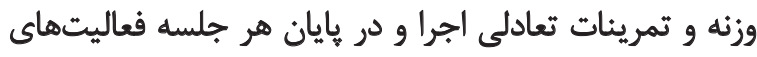
سردكردن انجام مىشد (جدول شماره Y و وَ).

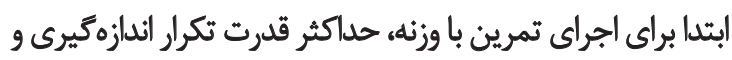

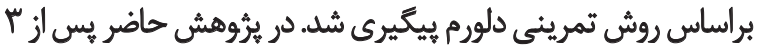

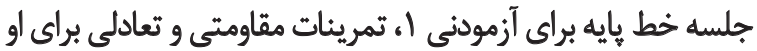

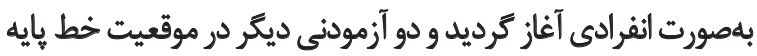

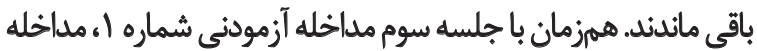

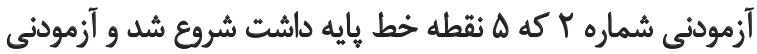

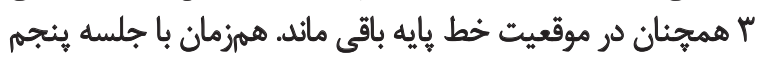

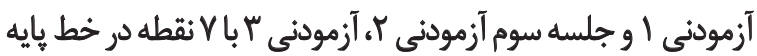

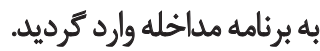

در اين بثروهش براى تجزيه و تحليل دادهها، ابتدا دادههاى خام

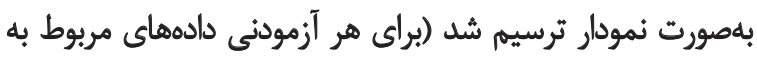

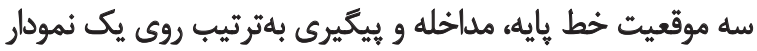

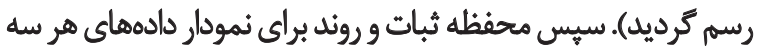

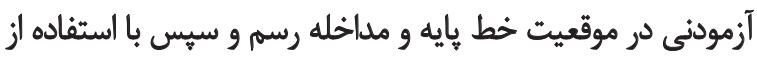

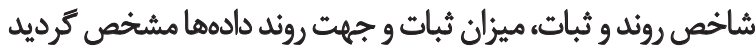

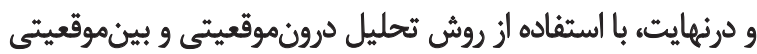

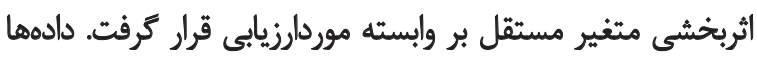

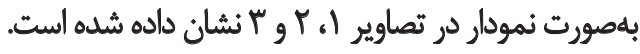

ياقتهها

نتايج تحليل ديدارى نمودارها نشان مى دهد تمرين مقاومتى و

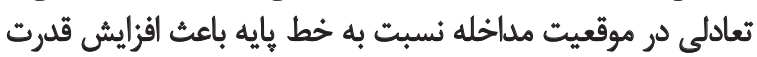

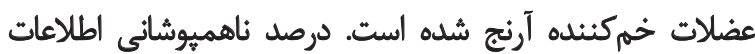

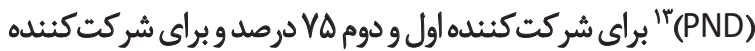
سوم . "ا درصد بهدست آمده است. جدول شماره ا،ويثخى فردى آزمودنى ها رانشان مى دهد. يافتههاى حاصل از تحليل ديدارى نمودار دادههاى هر سه آزمودنى نشاني

13. Percentage of Non-Overlapping Data
حال با توجه به اينكه مطالعات به تأثيرات تمرين مقاومتى بر قدرت

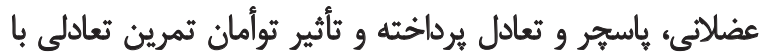

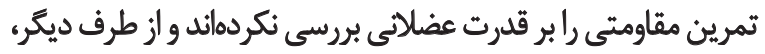

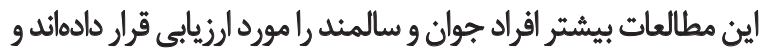

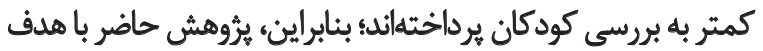

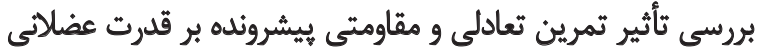
كودكان مبتلا به فلج مغزى طراحى و انجام يذيرفت.

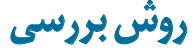

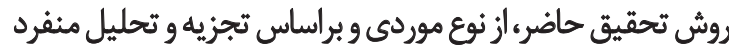

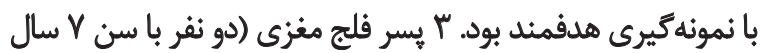

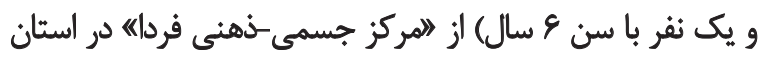

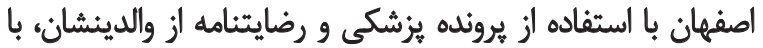
رعايت معيارهاى ورود و خروج به شرح زير انتخاب شدنداند

معيارهاى ورود: I- آزمودنى فلج مغزى Y-Y ساله؛ Y-Y جنسيت

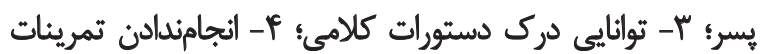

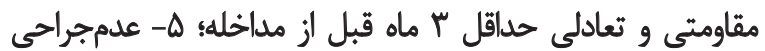

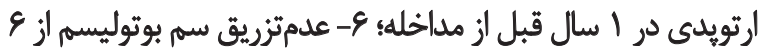

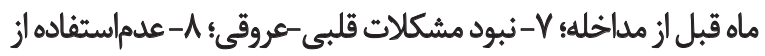
داروهاى آرامبخش در طول زمان مداخله.

معيارهاي خروج: 1 - عدم همكارى والدين و كودك؛ ب- وقون حوادث ارتويديك در طول زمان مداخله.

قدرت عضلات فلكسور آرنج و مهج دست توسط دستكاه دينامومتر

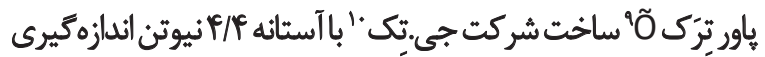

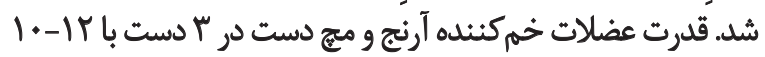

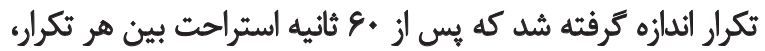

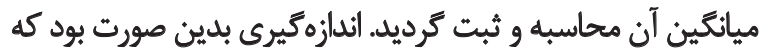

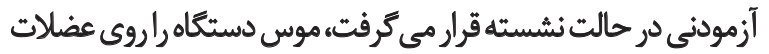

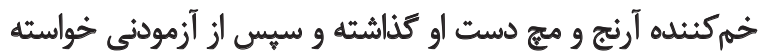

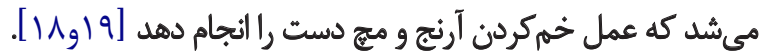

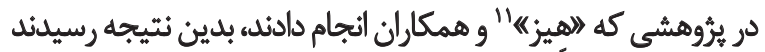

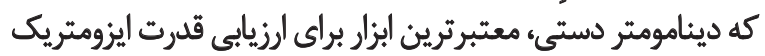

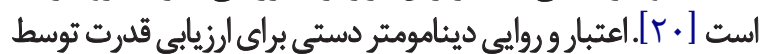

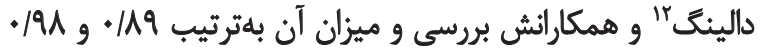

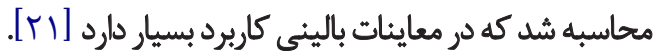

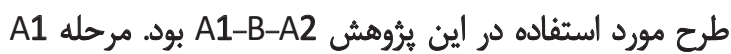

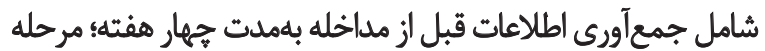

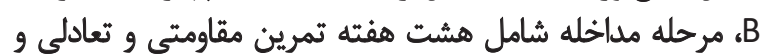

9. Power Track Õ

10. JTECK

11. Hayes

12. Dolling 
قاتدرت عضلات خممكنده آرنج آزمودنى شماره دو تغييرات افزايشى

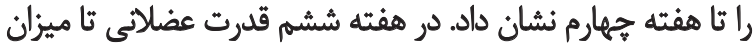

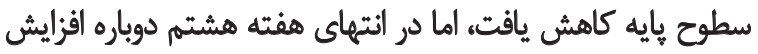

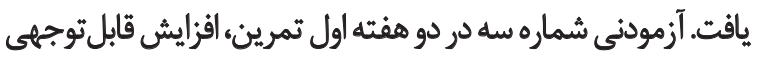

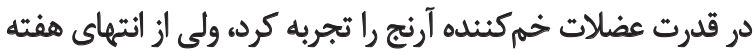

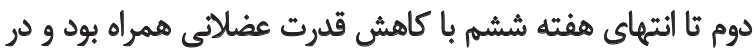
انتهاى هفته هشتم قدرت عضلانى افزايش رانشان دادي

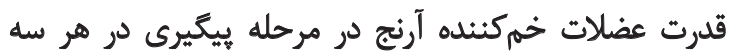

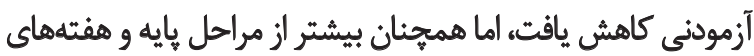

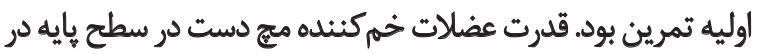

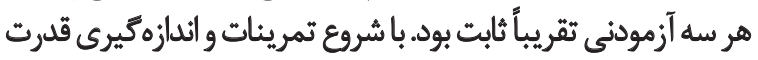

قدرت عضلات خمركنده ميج دست هر سه آزمودنى در موقعيت

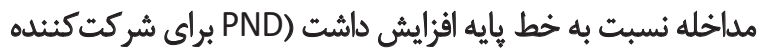

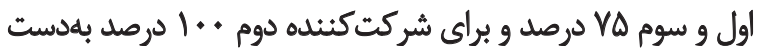
آمد). يافتهها در جداول شماره f و و ه نشان داده شده است.

ب

نتايج نشان مى دهد كه قدرت عضلات خمكنينده آرنج در مرحله

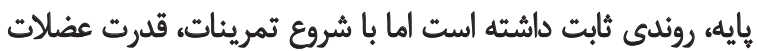

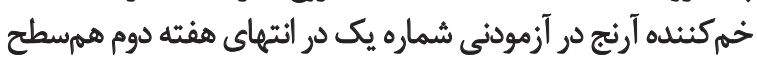

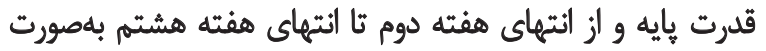

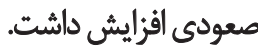

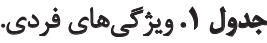

\begin{tabular}{|c|c|c|c|c|c|}
\hline آزمودنى & سن & جنسيت & وزن (كيلوكرم) & قد (ساتثىمثر) & توضيحات \\
\hline اول & 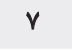 & 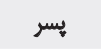 & WA & 11. & داى يلزُى اسياستيك \\
\hline دوم & $v$ & 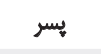 & r. & IIV & داي بالثى اتاكسيك \\
\hline سوم & 8 & 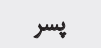 & ia & 1.8 & دائرّى اسياستيك \\
\hline
\end{tabular}

توانبخننى

جدول T. برنامه تمرين مقاومتى.

\begin{tabular}{|c|c|c|c|c|c|c|c|}
\hline \multicolumn{3}{|c|}{ شدت براساس درصد RM } & \multirow{2}{*}{$\frac{\text { تعداد تكرار }}{1 .}$} & \multicolumn{3}{|c|}{ (10ست } & \multirow{2}{*}{ خمركردن و بازكردن آرنج در هالت نشسيثه } \\
\hline 1.0 & va & Q. & & 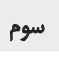 & دوم & 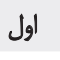 & \\
\hline $1 \ldots$ & va & a. & 1. & 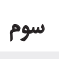 & دوم & 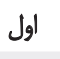 & خمكردن و بازٔكردن شائه در حالت نشسته \\
\hline $1 \ldots$ & va & ه. & 1. & 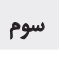 & 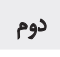 & الول & خمثردن و بازكردن هيج دست \\
\hline 1.0 & $V \Delta$ & ه. & 1. & 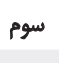 & دوم & 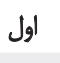 & دوركردن شائه در حالث نشسيثه \\
\hline 1.0 & Va & ఎ. & 1. & 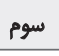 & دوم & 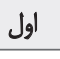 & تيزديك كردن شائه در حالت نُشسته \\
\hline
\end{tabular}

توانبخننى

جدول با. برنامه تمرين تعادلى.

\begin{tabular}{|c|c|}
\hline زمان & حركت \\
\hline 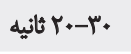 & تعادل روى توب فيزيوبال در حالت نشسته \\
\hline 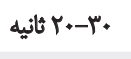 & قدمبرداشتن روى خط مسثقيم با حمايت \\
\hline 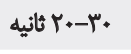 & ايستادن روى بياى راست و جبي با جشمان باز با حمايت ديوار \\
\hline 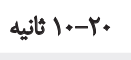 & إيستادن روى ياى راست و هب با جشمان باز با حمايت مربى \\
\hline 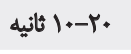 & ايستادن روى باى راست و هب با جشمان بسته با حمايت ديوار \\
\hline 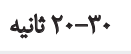 & الرسال و دريافت توب \\
\hline 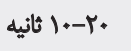 & شنا سوئدى با حمايت \\
\hline 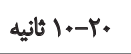 & 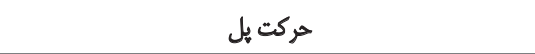 \\
\hline
\end{tabular}

توانبخننى 


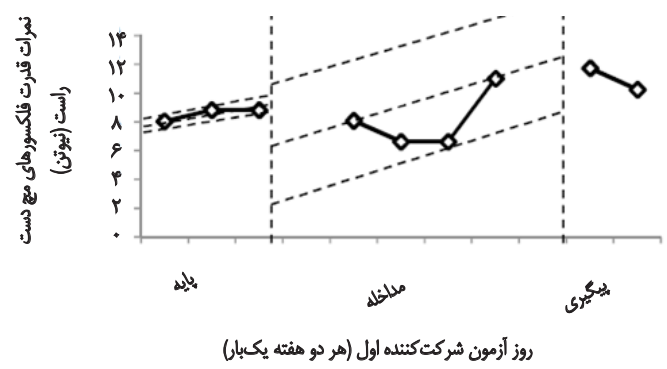

توانبخننى

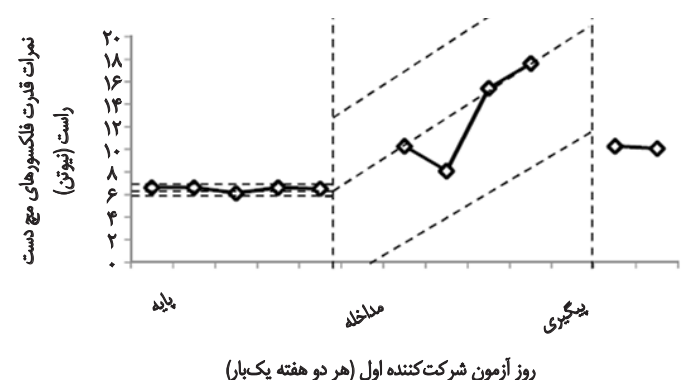

توانبخنتى

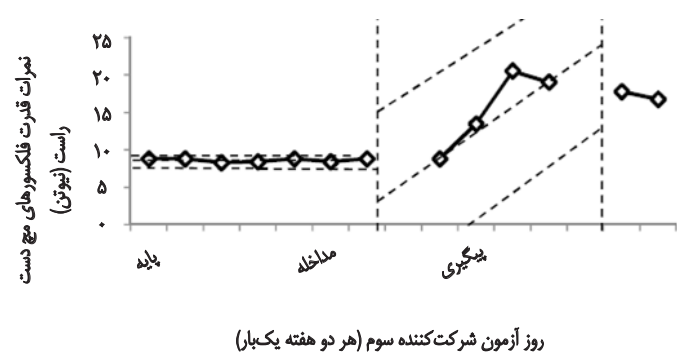

توانبخننى

شامل تكرار، شدت و طول مدت فعاليت براى كودكان فلج مغزى تورئ

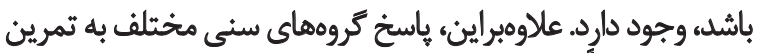

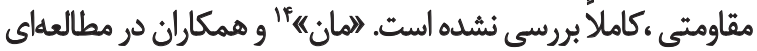

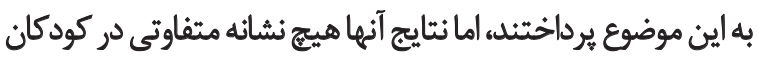

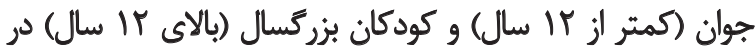

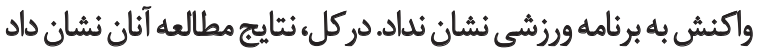

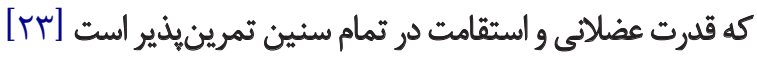

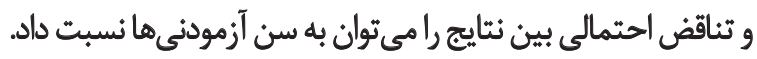

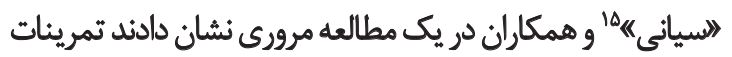

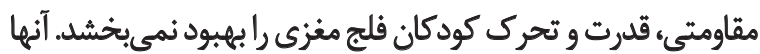

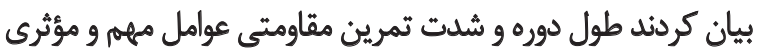

14. Mahon

15. Scianni

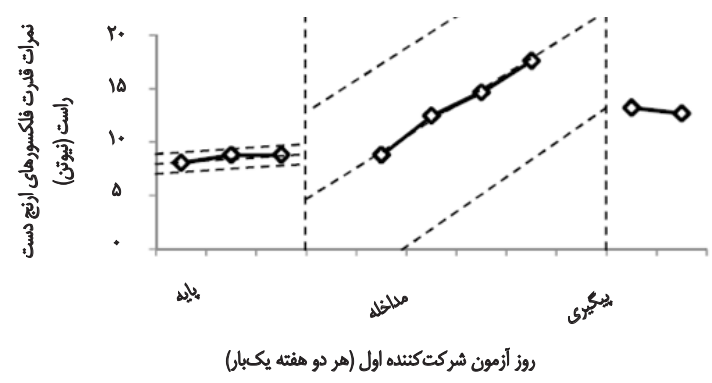

تصوير ا.قدرت عضلات خمكنيده آرنج و ميج دست آزمودنى اول.

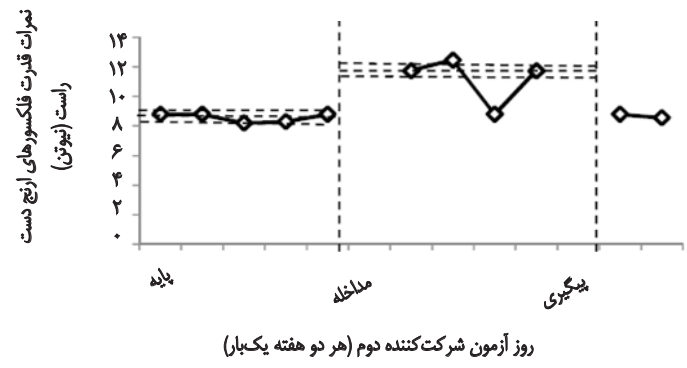

تصوير T. قدرت عضلات خمكنئله آرنج و ميج دست آزمودنى دوم.

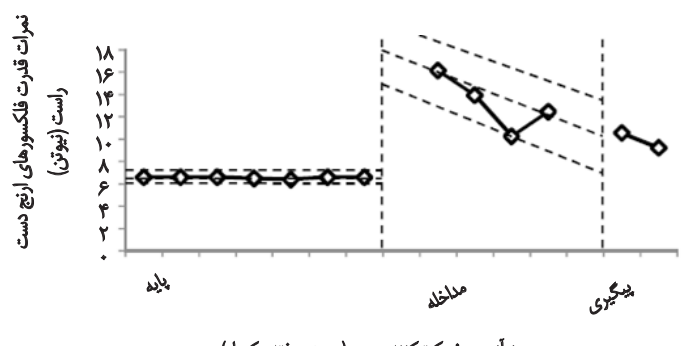

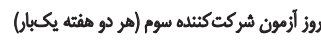

تصوير ب. قدرت عضلات خممننده آرنج و ميج دست آزمودنى سوم.

هر دو هفته يكبار نشان داد كه قدرت عضلات خممكننده ميج دست

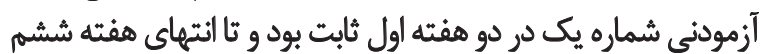

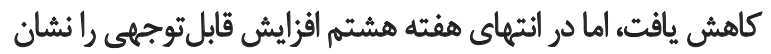

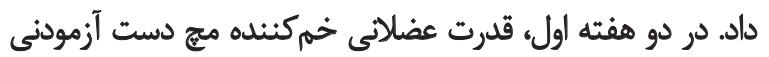

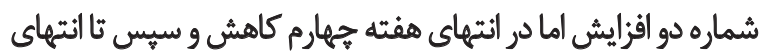

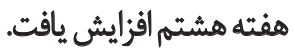
در آزمودنى شماره سه، با شروع تمرينات و تا انتهاى هفته ششم

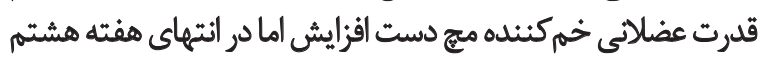

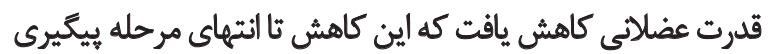

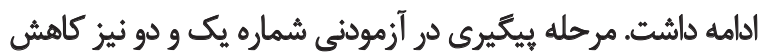

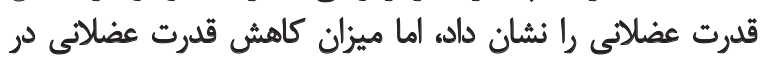
آزمودنى شماره دو بيشتر بود انشان تاكنون شواهد كمى براى شناسايى مطلوبترين برنامه ورزشي كه 
جدول f. جدول درونموقعيتى و بينموقعيتى براي خهمردن مفصل آرنج دست راست سه شركت كنيده.

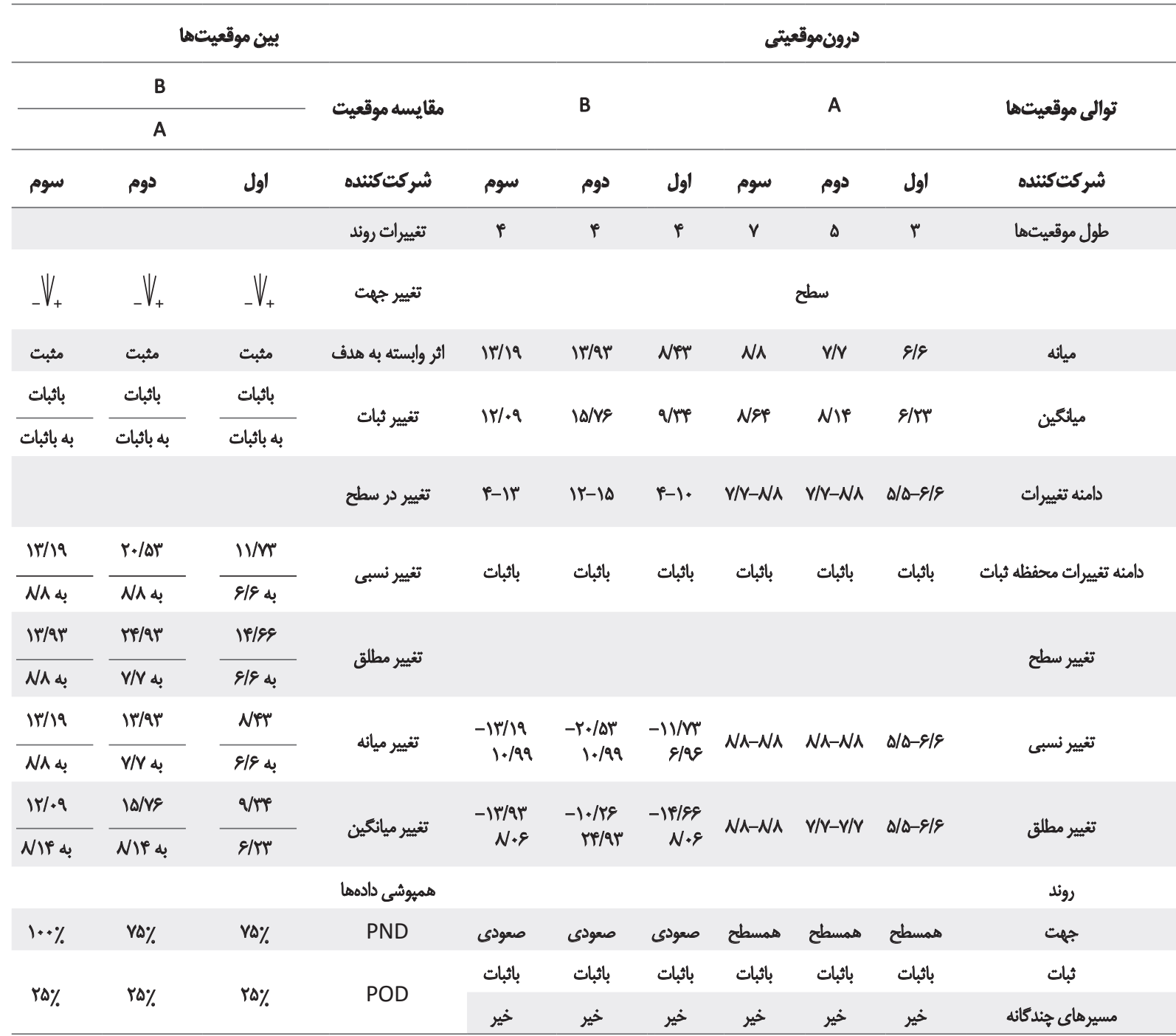

توانبخننى

ران و يلانتار فلكسور ميج يا تغيير معنادارى نداشت. نتايج آنها به

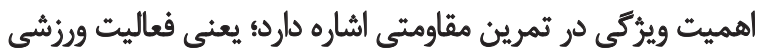

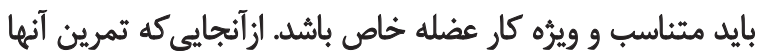

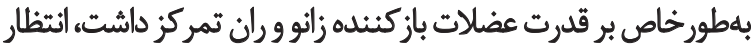
افززايش قدرت در اين عضلات وجود داشت [بات بازئ.

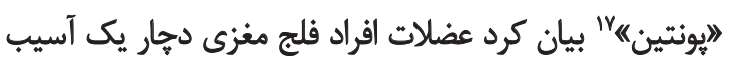

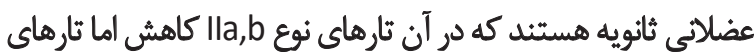

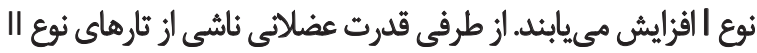

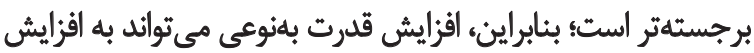

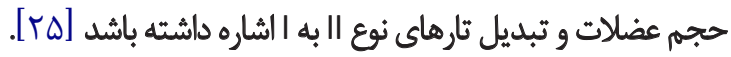

بر افزايش قدرت هستئد. بررسى آنها نشان داد مطالعات انجامشده با

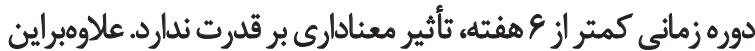

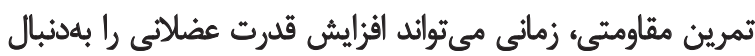

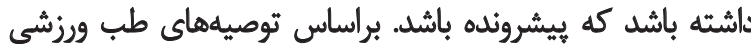

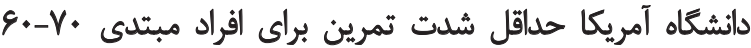

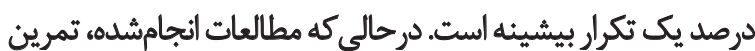

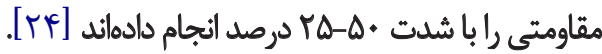

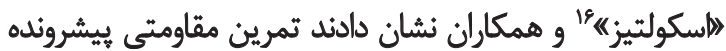

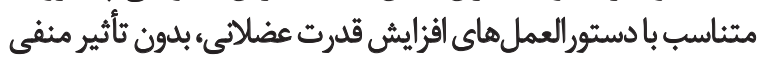

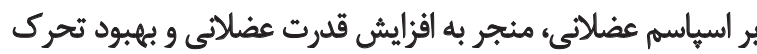

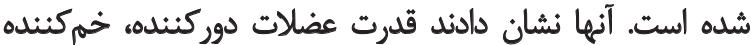


جدول ها. جدول درونموقعيتى و بينموقعيتى براى خمكردن مفصل هج دست سه شركت كنيده.

\begin{tabular}{|c|c|c|c|c|c|c|c|c|c|c|}
\hline \multicolumn{4}{|c|}{ بين موقعيتها } & \multicolumn{7}{|c|}{ درونموقعيتى } \\
\hline & B & 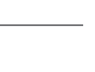 & مقايسه موقعيت & & B & & & A & & توالى موقعيتها \\
\hline \multirow[t]{2}{*}{ سوم } & دوم & اول & شركتكثنده & 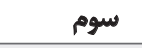 & ل دوم & اول & سوم & دوم & اول & شركت كثنده \\
\hline & & & تغييرات روند & $r$ & r & r & v & $\Delta$ & $r$ & طول موقعيتها \\
\hline$V_{+}$ & $-V_{+}$ & $V_{+}$ & تغيير جهت & \multicolumn{7}{|c|}{ سطح } \\
\hline مثبت & مثبت & هثبت & الثر وابسته به هـف & IE/TE & IYAT & $V / T T$ & NA & gis & NA & ميائه \\
\hline \multirow[t]{2}{*}{ به باثيثات } & به باثئبات & به باثئبات & تغيير ثبات & $10 / F$ & Ir/Ar & $N+8$ & NAI & E/FA & NDF & ميانكين \\
\hline & & & تغيير در سطح & $N A-r+/ \Delta T$ & $N \cdot 9-1 Y / 8$ & $8 / 8-11$ & $N r-N A$ & $9 / 1-8 / 9$ & $N \cdot r-N A$ & دامنه تغييرات \\
\hline$\frac{19 / \sqrt{9}}{N \wedge d 4}$ & $\frac{18 / 0}{9 / 8 d}$ & $\frac{N A}{N A d ب}$ & تغيير نسبى & باثبات & بائبات & باثيات & باثبات & بائبات & باثبات & دامنه تغييرات محفظه ثبات \\
\hline$\frac{19 \cdot 9}{N A d !}$ & $\frac{\mid V / 8}{9 / 8}$ & $\frac{11}{\text { NAd! }}$ & تغيير مطلق & & & & & & & تغيير سطح \\
\hline$\frac{19 / K 8}{\text { NAd }}$ & $\frac{\mid r / A r}{q / 9 \phi}$ & $\frac{V / \pi}{N A d \varphi}$ & تغيير هيانه & $11 / 1 r-12 / 9$ & $9 / \Delta-1 \% / 18$ & $V / T T-N A$ & $N B-N A$ & $\theta / \Delta-8 / 8$ & $N \cdot Y-N A$ & تغيير نسبى \\
\hline \multirow[t]{2}{*}{$\frac{\mid \Delta / \% F}{\text { Ne/ d! }}$} & 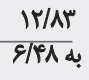 & $\begin{array}{l}\text { N.F } \\
\text { Nof }\end{array}$ & تغيير مياتكين & $N A-19 / \cdot 8$ & $1 . / T 8-1 V / 8$ & $N \cdot 9-11$ & $N A-N A$ & $8 / 0-8 / 8$ & $N \cdot r-N A$ & تغيير مطلق \\
\hline & & & هميوشى دادهها & & & & & & & روثل \\
\hline$V \Delta \%$ & $1.0 \%$ & $V \Delta \%$ & PND & صegra & صعودى & صeص & همسطح & همسطح & همسطح & جهت \\
\hline \multirow[t]{2}{*}{$r \Delta \%$} & $\%$ & $r \Delta \%$ & POD & باثبات & باثبات & باثبات & بائبات & كائبات & باثبات & ثبات \\
\hline & & & & خير & خير & خير & خير & خير & خير & مسير هائ جندكانه \\
\hline
\end{tabular}

توانبخننى

همسترينَ و جهارسر با حس عمقى را بررسى و كزارش كردند

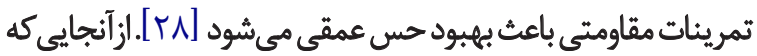

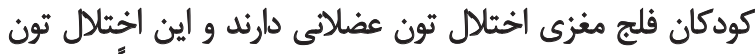

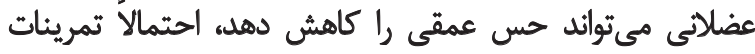

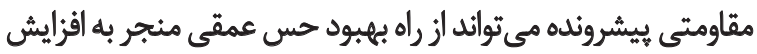
عملكرد كود كان فلج مغزى شود.

"إبكنى" "ن نشان داد تغييرات ناشى از تمرينات مقاومتى در افراد

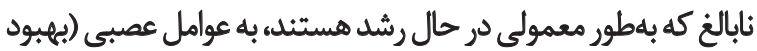

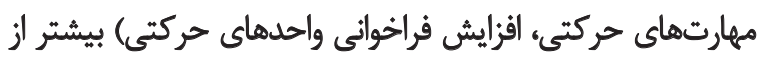

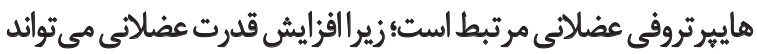

21. McNee
"كريكر "1" نشان داده است كه تمرينات مقاومتي بيشرونده

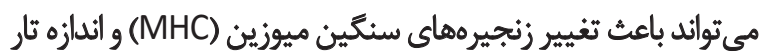

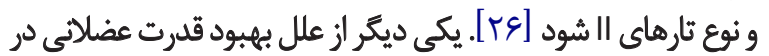

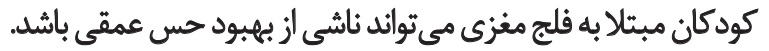

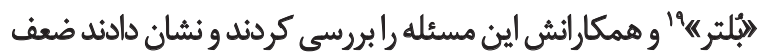

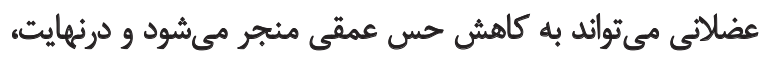

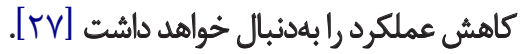

الايشيواكى 《'r و همكاران ارتباط كاهش قدرت عضلات

18. Kryger

19. Bulter

20. Nishiwaki 
ناشى از افزايش حجم عضلاتى رابهدنبال داشته باشد. تعداد تكرار اهايى

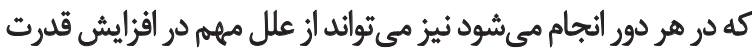

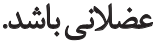

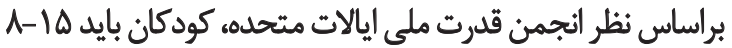

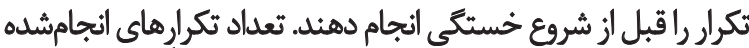

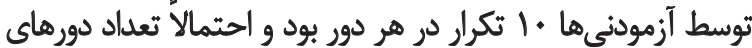

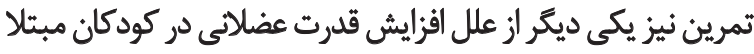

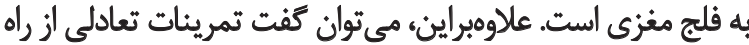

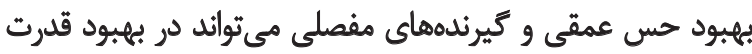
عضلانى در كودكان مبتلا به فلج مغزى مؤثر باشد.

افراد مبتلا به فلج مغزى، با مشكلات زيادى مواجه هستند. يكى از از

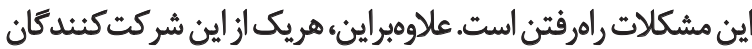

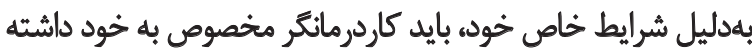

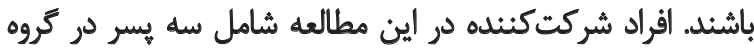

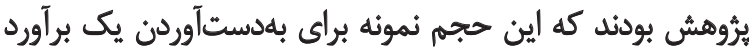
ابتدايي، كافى بلنظر مئرسد.

\section{نتيجليرى}

عبروهش حاضر نشان داد تمرينات مقاومتى و تعادلى بر قدرت

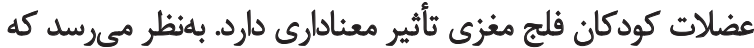

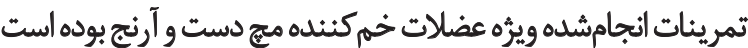

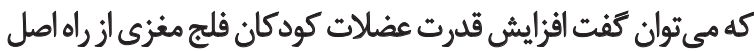

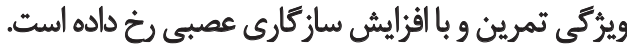
يكى از نكات مهام در مؤثربودن تمرين مقاومتى، شدت تمرين

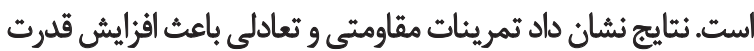

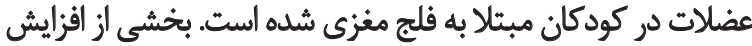

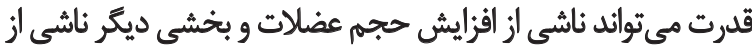

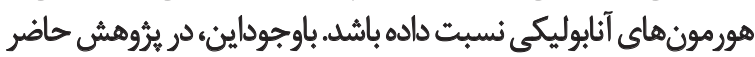

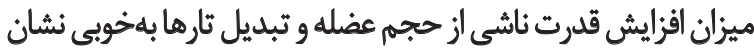

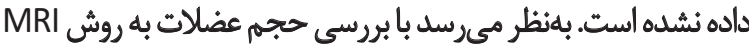

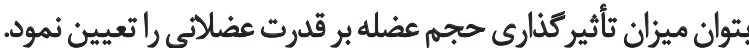

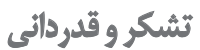

اين يُروهش در المركز جسمى -ذهنى فرداها در شهر اصفهان

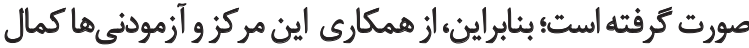
تشكر و قدردانى بهعمل آورده مي شئود.
در غياب هايبرتروفى رخ دهد [ع| [آس آسيبشناسى اوليه فلج مغزى عاري

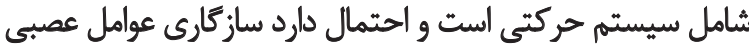

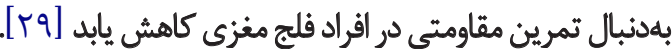

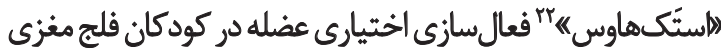

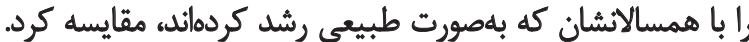

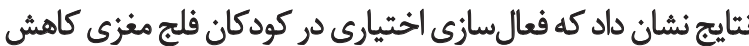

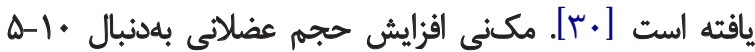

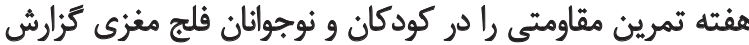

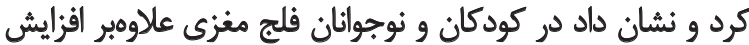

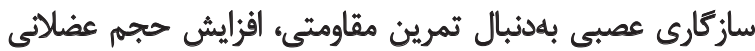

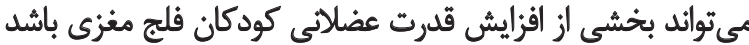

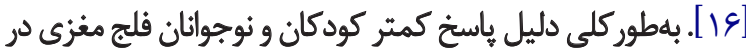

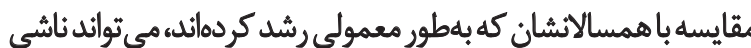

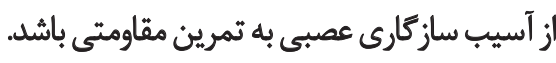

آنجهه آشكار بهنظر مىرسد اين است كه مدت تمرين بايد بهاندازه

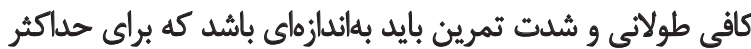

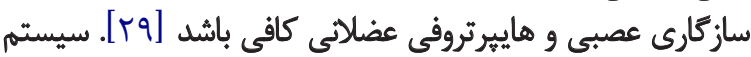

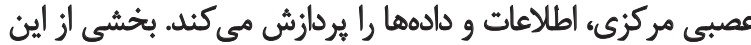

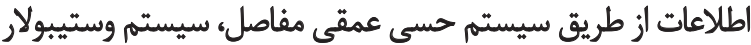

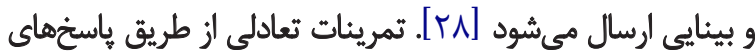

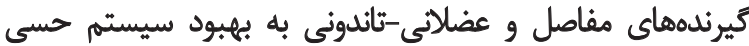

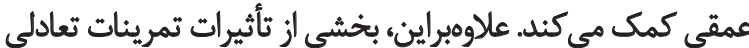

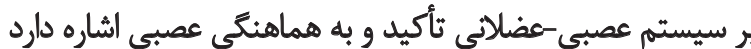

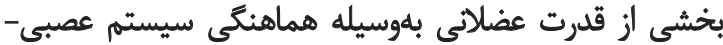

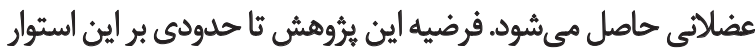

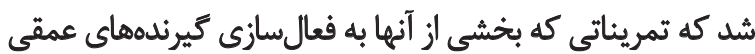

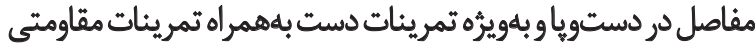
به بهبود فزاينده كمك خواهد كرد.

در مطالعات قبلى كزارش مار نشان داد زمانى كه شدت تمرين بين

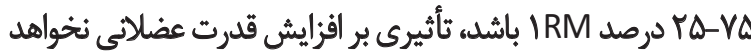

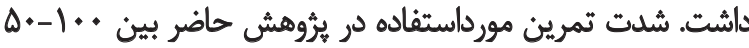

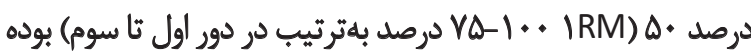

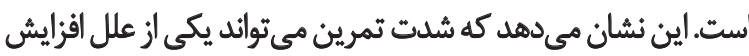
نيروى عضلاتى در كودكان مبتلا به فلج مغزى باشد.

يكى ديكر از عوامل مؤثر بر افزايش قدرت عضلات كودكان مبتلا

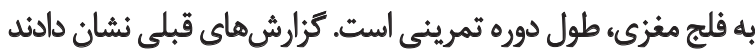

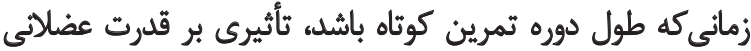

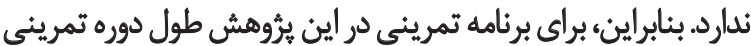

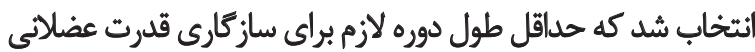




\section{References}

[1] Aisen ML, Kerkovich D, Mast J, Mulroy S, Wren TA, Kay RM, et al. Cerebral palsy: clinical care and neurological rehabilitation. Lancet Neurology. 2011; 10(9):844-52.

[2] Odding E, Roebroeck ME, Stam HJ. The epidemiology of cerebral palsy Incidence, impairments and risk factors. Disability and Rehabilitation. 2006; 28(4):183-191.

[3] Scholtes VA, Becher JG, Comuth A, Dekkers H, van Dijk L, Dallmeijer AJ. Effectiveness of functional progressive resistance exercise strength training on muscle strength and mobility in children with cerebral palsy: a randomized controlled trial. Developmental Medicine \& Child Neurology. 2010; 52(6):107-113

[4] Ross SA, Engsberg JR. Relationships between spasticity, strength, gait, and the GMFM-66 in persons with spastic diplegia cerebral palsy. Archives of Physical Medicine and Rehabilitation. 2007; 88(9):1114-20.

[5] Damiano DL, Dodd K, Taylor NF. Should we be testing and training muscle strength in cerebral palsy? Developmental Medicine \& Child Neurology. 2002; 44(1):68-72.

[6] Fowler EG, Ho TW, Nwigwe AI, Dorey FJ. The effect of quadriceps femoris muscle strengthening exercises on spasticity in children with cerebral palsy. Physical Therapy. 2001; 81(6):1215-1223.

[7] Faigenbaum AD, Kraemer WJ, Blimkie CJ, Jeffreys I, Micheli LJ, Nitka $\mathrm{M}$, et al. Youth resistance training: updated position statement paper from the national strength and conditioning association. Journal of Strength \& Conditioning Research. 2009; 23(5):60-79.

[8] Shumway-Cook A, Hutchinson S, Kartin D, Price R, Woollacott M. Effect of balance training on recovery of stability in children with cerebral palsy. Developmental Medicine \& Child Neurology. 2003; 45(9):591-602

[9] Morton JF, Brownlee M, McFadyen AK. The effects of progressive resistance training for children with cerebral palsy. Clinical Rehabilitation. 2005; 19(3):283-9

[10] Eek MN, Tranberg R, Zugner R, Alkema K, Beckung E. Muscle strength training to improve gait function in children with cerebral palsy. Developmental Medicine \& Child Neurology. 2008; 50(10):759-64

[11] Lee JH, Sung IY, Yoo JY. Therapeutic effects of strengthening exercise on gait function of cerebral palsy. Disabilty and Rehabilitation. 2008; 30(19):1439-44.

[12] Dodd KJ, Taylor NF, Graham HK. A randomized clinical trial of strength training in young people with cerebral palsy. Developmental Medicine \& Child Neurology. 2003; 45(10):652-7.

[13] Liao HF, Liu YC, Liu WY, Lin YT. Effectiveness of loaded sit-tostand resistance exercise for children with mild spastic diplegia: a randomized clinical trial. Archives of Physical Medicine and Rehabilitation. 2007; 88(1):25-31

[14] Abdolvahab M, Abbasi S, Hadian MR, Jalili M, Jalaei S. [Effects of Progressive Resistive Exercise on isometric strength o shoulder extensor and abductor muscles in adult hemiplegic (Persian)]. Joutnal of Modern Rehabilitation. 2009; 3(3-4):30-24.

[15] Azzam AM. Effect of Hand Function Training on Improvement of Hand Grip Strength in Hemiplegic Cerebral Palsy in Children. Novel Physiotherapies. 2012; 2(6):5-2.
[16] McNee AE, Gough M, Morrissey MC, Shortland AP. Increases in muscle volume after plantarflexor strength training in children with spastic cerebral palsy. Developmental Medicine \& Child Neurology. 2009; 51(6):429-435

[17] Burney H, Taylor N, Dodd K. A qualitative analysis of the benefits of strength training for young people with cerebral palsy. Developmental Medicine \& Child Neurology. 2003; 45(10):658-63.

[18] Kendall FP, McCreary EK. Province PG.Muscles testing and function. Philadelphia: Lippincott Williams and Wikins; 1983, pp. 215-279.

[19] Daniels L, Worthingham C. Muscle testing: Techniques of Manual Examination. Philadelphia: W.B. Saunders Company; 2002, p. 107-138.

[20] Hayes K, Walton JR, Szomor ZL, Murrell GA, et al. A randomised clinical trial evaluating the efficacy of physiotherapy after rotator cuff repair. Australian Journal of Physiotherapy. 2002; 50(2):77-83.

[21] Dollings H, Sandford F, O'Conaire E, Jeremy S. Shoulder strength testing: The intra-and inter-tester reliability of routine clinical tests, using the PowerTrack ${ }^{\mathrm{TM}}$ II Commander. Shoulder and Elbow. 2012 4(2):131-140.

[22] Cipani E. Practical Research Methods for Educators. New York: Springer Publishing Company; 2009.

[23] Mahon AD. Exercise training. In: Armstrong N, van Mechelen W, editors. Paediatric Exercise Science and Medicine. New York: Oxford University Press; 2000, p. 201-11

[24] Scianni A, Jane M Butler, Louise Ada and Luci F Teixeira-Salmela. Muscle strengthening is not effective in children and adolescents with cerebral palsy: A systematic review. Australian Journal of Physiotherapy. 2009; 55(2):81-87.

[25] Ponten E, Friden J, Thornel L, Lieber R. Spastic wrist flexors are more severly affected than wrist extensors in children palsy. Developmental Medicine \& Child Neurology. 2005; 47(6):348.

[26] Kryger I, Andersen L. Resistance training in the oldest old: cosequences for muscle strength, fiber, fiber size, and MHC isoforms. Scandinavian Journal of Medicine \& Science in Sports. 2007; 17(4):422-430.

[27] Butler A, Lord S, Rogers M, Fitzpatrick R. Muscle Weakness impairs the proprioceptive control of human standing. Brain Research .2008 1242: 244-251.

[28] Nishiwaki GA, Tanaka K, Urabe Y. The effect of muscle strength on proprioceptive function after anterior cruciate ligament reconstruction of the knee. Japanese Journal of Physical Fitness and Sports Medicine. 2007; 56(5):451-460

[29] Verschuren O, Louise A, Désirée BM, Gorter JW, Scianni A, Ketelaat M. Future resistance training protocols with spastic cerebral palsy: considerations for muscle strengthening in children and adolescents. Physical Therapy. 2011; 91(7):1130-1139.

[30] Stackhouse SK, Binder-Macleod SA, Lee SC. Voluntary muscle activation, contractile properties, and fatigability in children with and without cerebral palsy. Muscle Nerve. 2005; 31(5):594-601. 